\section{Dendritic cells in the gut: to sample and to exclude?}

\author{
C Nicoletti ${ }^{1}, M$ Regoli $^{2}$ and \\ E Bertelli ${ }^{2}$
}

${ }^{1}$ Laboratory of Mucosal Immunology, Integrated

Program of Gl Tract Biology, Institute of Food

Research, Norwich, UK. ${ }^{2}$ Department of

Pharmacology "G. Segre," University of Siena, Italy.

Correspondence: C Nicoletti (claudio.nicoletti@

bbsrc.ac.uk)

To the editor: Over the past few years one of the most important concepts that emerged in the area of gut immunology derived from the observation by Rescigno et al. ${ }^{1}$ on the ability of intestinal dendritic cells (DCs) to extend cellular extensions between epithelial cells into the intestinal lumen, to internalize bacteria and shuttle them across the epithelial barrier. This sophisticated mechanism for antigensampling complements the well-studied M-cell-mediated transport of particulate antigen and bacteria. ${ }^{2}$ We have recently reported that DC-mediated sampling is not the only event taking place at the host-pathogen interface in the small intestine during the early stage of bacterial infection. Indeed, a proportion of DCs that rapidly migrate to the lamina propria following challenge with non-invasive Salmonella lacking the Salmonella Pathogenicity Island 1 ( $\Delta$ SPI1) did not act as an antigen-sampling cell but crossed the epithelium and moved into the gut lumen (Figure 1) before or following internalization of Salmonella. ${ }^{3}$ The

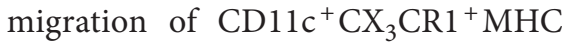
$\mathrm{II}^{+} \mathrm{CD} 11 \mathrm{~b}^{-} \mathrm{CD} 8 \alpha^{-} \mathrm{DCs}$ was found to be flagellin- and MyD88-dependent, it was restricted to the small intestine and it was not observed in MyD88 mice. Interestingly, intraluminal DCs internalized Salmonella but did not cross

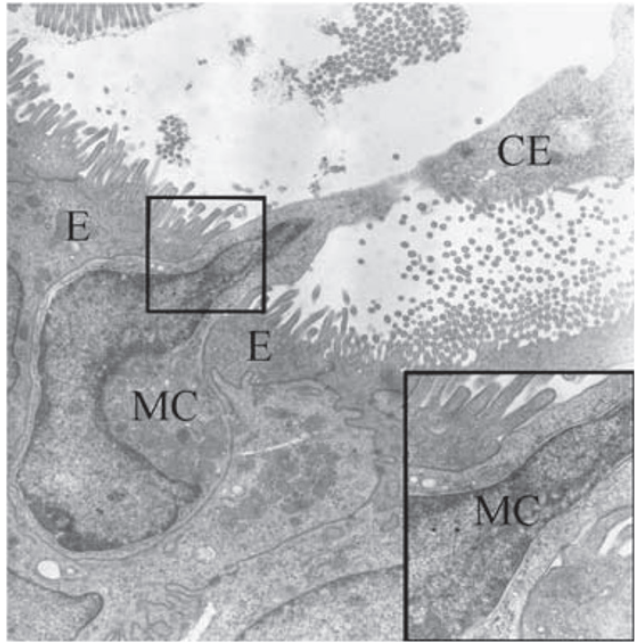

Figure 1 TEM micrographs showing an immune cell embedded between epithelial cells moving into the intestinal lumen with a large portion of the body already inside the lumen following challenge with non-invasive $\Delta$ SPI1 Salmonella. Inset: Details of the contact between the migrating cell and the adjacent epithelial cells. ( $\mathrm{MC}=$ migrating cell; $\mathrm{E}=$ epithelial cell; $\mathrm{CE}=$ cellular extension).

the epithelium to return into the tissue. The finding that DCs migrate into the gut lumen following a challenge with a pathogen suggests a few observations. First, it appears that the same antigenic stimulus (e.g., non-invasive Salmonella) can induce DCs to either sample or to migrate into the gut lumen thus showing the complexity of the signaling network operating at the host-pathogen interface; second it tells us that many of the intraepithelial DC extensions previously considered to be "sampling" devices are a feature of DCs undergoing transepithelial migration. At this time the role of the intraluminal "bacteria-capturing" DCs remains to be determined. The migration of phagocytes into the gut lumen was described in the past and it was interpreted as a mechanism of cellular control of the gut pathogens; ${ }^{4,5}$ thus, it is possible to hypothesize that DCs are also part of a similar defence mechanism. Sending phagocytes, including DCs, into the lumen of the small intestine would possibly help to limit the number of pathogens that can cross the epithelial barrier and infect the host; a strategy that would complement the immune exclusion mediated by mucous and sIgA antibody.

\section{DISCLOSURE}

The authors declared no conflict of interest.

C 2009 Society for Mucosal Immunology

\section{REFERENCES}

1. Rescigno, M. et al. Dendritic cells express tight junction proteins and penetrate gut epithelial monolayers to sample bacteria. Nat. Immunol. 2, 361-367 (2001).

2. Nicoletti, C. Unsolved mysteries of intestinal M cells. Gut 47, 735-739 (2000).

3. Arques, J.L. et al. Salmonella induced flagellin- and MyD88-dependent migration of bacteria-capturing dendritic cells into the gut lumen. Gastroenterology; e-pub ahead of print (2009).

4. Bellamy, J.E.C. \& Nielsen, N.O. Immunemediated emigration of neutrophils into the lumen of the small intestine. Infect. Immun. 9, 615-619 (1974).

5. Regoli, M., Borghesi, C., Bertelli, E. \& Nicoletti, C. A morphological study of the lymphocyte traffic in Peyer's patches after an in vivo antigenic stimulation. Anat. Rec. 239, 47-54 (1994).

doi:10.1038/mi.2009.92 